\title{
Tangence
}

\section{Le goût du luxe}

Asianisme et bonne chère à l'âge classique

The taste for luxury

Asianism and good food at the Classical age

\section{Frédéric Charbonneau}

Numéro 65, hiver 2001

Figures de l'Orient

URI : https://id.erudit.org/iderudit/008226ar

DOI : https://doi.org/10.7202/008226ar

Aller au sommaire du numéro

Éditeur(s)

Presses de l'Université du Québec

ISSN

0226-9554 (imprimé)

1710-0305 (numérique)

Découvrir la revue

Citer cet article

Charbonneau, F. (2001). Le goût du luxe : asianisme et bonne chère à l'âge

classique. Tangence, (65), 23-32. https://doi.org/10.7202/008226ar d'utilisation que vous pouvez consulter en ligne.

https://apropos.erudit.org/fr/usagers/politique-dutilisation/ 


\section{Le goût du luxe. \\ Asianisme et bonne chère à l'âge classique Frédéric Charbonneau, Université McGill}

Dans un texte célèbre mettant aux prises Socrate et le rhéteur Gorgias, Platon opère un rapprochement d'autant plus efficace qu'il est inattendu. La rhétorique, écrit-il, est comme la cuisine, l'une "correspond, pour l'âme, à ce qu'est [l'autre] pour le corps": ce sont des procédés que la flatterie a inventés pour singer des techniques utiles. Or

du bien, elle n'a nul souci, mais, par l'attrait du plaisir, elle tend un piège à la sottise qu'elle abuse, et gagne de la sorte la considération. C'est ainsi que la cuisine contrefait la médecine et feint de connaitre les aliments qui conviennent le mieux au corps, de telle façon que si des enfants, ou des hommes aussi déraisonnables que des enfants, avaient à juger, entre le médecin et le cuisinier, lequel connaît le mieux la qualité bonne ou mauvaise des aliments, le médecin n'aurait qu'à mourir de faim. [...]

Pour abréger, je te dirai [...] que la sophistique est à la législation comme la toilette est à la gymnastique, et que la rhétorique est à la justice comme la cuisine est à la médecine ${ }^{1}$.

Cette condamnation allait longtemps peser sur des plaisirs jugés indignes, au gré du philosophe, d'hommes adultes et libres, trompés par là et voués à l'univers sensible. L'idéalisme méfiant face aux éblouissements du corps y rejoignait l'austère doctrine selon laquelle le vrai se passe des charmes d'une éloquence apprise. La métaphore du procès renforce, chez Platon, ce parallèle entre le cuisinier et le rhéteur: si l'un comme l'autre sait gagner le public à sa cause, c'est qu'ils le séduisent et le dupent, flattant ses goûts, ses préjugés, donnant l'agrément pour le bien, les blandices pour la vérité. "Des mets et des mots", titre d'un bel essai de Michel Jeanneret ${ }^{2}$, le destin parallèle nous occupera ici, et les

1. Platon, Gorgias, éd. J.-Fr. Pradeau, trad. A. Croiset, Paris, Les Belles Lettres, 1997, p. 56-61.

2. Michel Jeanneret, Des mets et des mots. Banquets et propos de table à la Renaissance, Paris, José Corti, 1987. 
soupçons dont ils ont fait l'objet à l'aune d'une morale qui ne veut que le pur et en regard de laquelle tous deux paraissent trop corruptibles. En effet, chacune de ces pratiques éclaire l'autre, et le recours à des concepts communs pour les appréhender témoigne de la profondeur historique de leur lien: "routines" dépourvues de fondement solide, elles tendent toutes deux naturellement vers l'ornement qui dissimule ou qui maquille leur indigence de fait, luxe du verbe ou du ragoût, illusoire abondance à laquelle on doit opposer la sobre rigueur du savoir.

Avec le développement des Belles Lettres à la Renaissance puis à l'âge classique, la rhétorique, on le sait, est pourtant devenue la pierre angulaire de ce qui sera plus tard nommé littérature. Elle est aussi l'une des bases de l'enseignement et, à ce titre, elle a pour fonction de nourrir l'écolier, au sens que revêtait anciennement ce verbe, qui conservait l'ambiguïté du nutrire latin : à la fois alimenter et éduquer ${ }^{3}$. L'éloquence civilisatrice traverse d'un même souffle la chaire et le barreau, l'histoire et la poésie; écoles, traités et débats fourmillent au sein de la République des Lettres. Parmi les sujets de litige, il en est un tout particulièrement qui retiendra notre attention, et qui oppose deux esthétiques mais aussi deux morales: l'atticisme et l'asianisme. Les termes disent assez l'origine grecque de ce conflit. L'atticisme, c'est l'ancien style des orateurs d'Athènes, l'asianisme, celui des peuples orientaux; d'un côté la pureté, la clarté, la sévérité; de l'autre la suavité, l'ornementation, l'abondance.

En 1608, Henri Dupuy, disciple et successeur de Juste Lipse à Louvain, publie un essai en latin intitulé De laconismo syntagma dans lequel il défend une "philosophie générale de l'atticisme". Il y a selon lui, note Marc Fumaroli dans L'âge de l'éloquence,

deux grandes familles [d'esprits] dont l'incompatibilité domine l'histoire de la res literaria. L'une, liée au luxe, au désordre des mours, à l'effémination des caractères, à la demi-culture, favorise un style abondant et périodique, riche de "mots" inutiles et vains, et pauvres de "choses". [...] L'autre famille, liée à la pureté des mours, à la santé, à la frugalité, à la sobriété, à la chasteté, à la virilité enfin, indissociable de la vigueur intellectuelle et de la vraie science, s'exprime tout naturellement en un style bref, à la fois efficace et irrésistible, pauvre de "mots" et riche de "choses" solides. Ces deux familles en conflit expliquent

3. Michel Jeanneret, ouvr. cité, p. 126. 
l'histoire de l'Éloquence: à l'origine était la brièveté, dont l'Athènes classique fit un art, l'atticisme qui résumait l'Éloquence; fascinée par celle-ci, les peuples d'Asie, ignorants et corrompus, en inventèrent une version dégénérée, l'asianisme, qui contamina Athènes $[\ldots]^{4}$.

Chez ce grand humaniste, le reproche adressé par Platon à toute rhétorique est reporté sur le seul style "asiatique": ars dicendi sensuel et vain, nourrissant l'attrait des plaisirs faciles, du luxe et de la mollesse; l'atticisme, au contraire, est chaste, vigoureux, frugal. J'insiste sur ce dernier mot, qui semble retenir encore l'antique comparaison. Or précisément, en cette même année 1608, Dupuy publie un second opuscule contre le luxe des repas, le De Conviviorum Luxu Epistola, rapidement augmenté et remis sous presse coiffé d'un nouveau titre: Comus, sive Phagesiposia cimmeria, c'est-à-dire Comus, Ou banquet dissolu des Cimmeriens, titre de la version française donnée cinq ans plus tard par Nicolas Pelloquin ${ }^{5}$. Peuple originaire du Caucase qui déferla sur l'Asie Mineure au $\sim \mathrm{VIII}^{\mathrm{e}}$ siècle et fut repoussé à Éphèse, les Cimmériens étaient des barbares de l'Est; cuisine ou rhétorique, c'est tout un: le luxe corrupteur nous vient d'Orient.

Dans ce traité en forme d'allégorie, Dupuy nous représente le dieu de la bonne chère sinistrement accompagné: le "luxe", la "lasciveté ", la "repletion ", l' yvrongne-rie" lui font escorte.

Proche estoient la folie, la fureur, les noises, les debats, \& les bateries : \& puis le vomissement, la crudité, \& les maladies,

Et les subites morts, la vieillesse intestate.

[...]. Ce Comus est l'entretien des vices; il prend les esprits en ses delices, pour les énerver. Ce luxe que vous voyez, \& ceste lasciveté, c'est une ruine, \& qui esteint en l'homme, tout ce qu'il y a de genereux. Que pensez vous du reste de cette trouppe, molle, \& effeminée? [...] Comus a voulu bannir la candeur, \& la franchise, \& a reçeu chez soy la feinte, \& la tromperie ${ }^{6}$.

4. Marc Fumaroli, L'âge de l'éloquence. Rhétorique et "res literaria" de la Renaissance au seuil de l'époque classique, [1980], Paris, Albin Michel, 1994, p. 159.

5. Henri Dupuy, Comus, Ou banquet dissolu des Cimmeriens. Songe. Où par une infinité de belles feintes, gayes, gentilles, \& serieuses inventions, les moeurs dépravées de ce siecle (E principalement aux banquets) sont doctement, naïvement, \& singulierement décrites, reprises, E condamnées, traduit du latin d'Erycius Puteanus par Nicolas Pelloquin, Paris, Gilles Robinot, 1613.

6. Henri Dupuy, ouvr. cité, f $\mathrm{f}^{\circ} 11,22 \mathrm{v}^{\circ}-23 \mathrm{r}^{\circ}$. 
Les paradigmes sont les mêmes dans les deux ouvrages: d'un côté, l'Orient dégénéré, immoral, fourbe, spécieux; de l'autre, l'Occident détenteur de vertus naïves, de vertus d'ancienne roche, à présent menacées par Babylone. L'abondance, le superflu qui masque un vide caché comme une tare, l'enflure de la copia éructant mets et mots par sa corne, tout cela suggère une sorte d'envahissement qui appelle en retour la guerre sainte - car l'Oriental à l'époque, c'est aussi l'infidèle, le Turc dont les armées sont aux portes de Vienne.

Mais sans doute s'agit-il avant tout chez Dupuy d'une guerre intérieure et d'une lutte contre la tentation. La perspective chrétienne, en effet, en résonance avec le platonisme, détermine aussi bien la critique de la table que celle de la rhétorique. Ainsi, dans le De doctrina christiana, saint Augustin rappelait aux orateurs que les mots et les choses terrestres ne sont que les signes des choses divines, se livrant "du même coup à une critique implicite de l'ornatus païen, leurre qui reflète celui du monde sensible ${ }^{7}$ ". C'est pourquoi il avait plaidé pour un style clair que n'offusquerait nulle surcharge; il évitait ainsi l'écueil de la delectatio, qui consiste à jouir de la créature en oubliant le Créateur. Une attitude semblablement pernicieuse est à l'œuvre dans la gourmandise, flétrie par Bossuet à la suite de l'évêque d'Hippone au début du Traité de la concupiscence. La sagesse du Créateur, qui nous force à nous soutenir

par la douleur violente de la faim et de la soif et par les defaillances insupportables qui les accompaignent, nous y invite encore par le plaisir qu'elle a attaché aux fonctions naturelles de boire et de manger [...] Mais les hommes ingrats et charnels ont pris occasion de ce plaisir pour s'attacher a leur corps plustost qu'a Dieu, qui l'avoit fait et ne cessoit de le sustenter par des moyens si agreables. Le plaisir de la nourriture les captive: au lieu de manger pour vivre, ils semblent, comme disoit cet ancien et apres luy saint Augustin, ne vivre que pour manger ${ }^{8}$.

La vigilance de la foi doit servir de rempart contre le luxe impie; et l'Orient dont elle protège se tapit en chacun de nous.

Il appartiendra au XVIII ${ }^{\mathrm{e}}$ siècle de modifier cette optique traditionnelle. Le statut du luxe, et particulièrement sous ses aspects

7. Marc Fumaroli, ouvr. cité, p. 71.

8. Bossuet, Traité de la concupiscence, éd. Ch. Urbain et E. Levesque, Paris, Les Belles Lettres, 1930, chap. iv, p. 10. 
matériels, y devient en effet l'enjeu d'un important débat d'économie politique. Du III $^{\mathrm{e}}$ siècle avant l'ère chrétienne jusque vers 1650, le souci du législateur semble être demeuré le même à cet égard: pour s'en tenir au seul domaine de la table, déjà la République romaine avait tenté d'en interdire les excès en multipliant les lois somptuaires (par exemple la célèbre Lex Fannia, en 161 av. J.-C.) qui fixaient aussi bien le nombre des convives que les sommes investies dans les festins. De même en France, les édits s'étaient succédés de Philippe le Bel à Louis XIII dans le but de régler le luxe des repas. En 1705, Nicolas Delamare, commissaire au Châtelet, détaille dans son Traité de la police un édit de janvier 1629 qui défendait à toutes personnes "d'user au service de leurs tables, pour quelque pretexte \& occasion que ce soit, même aux festins de nôces \& de fiançailles de plus de trois services en tout"; d'y mettre "plus de six pieces au plat, soit de boüilly ou de rôty, de quelque sorte de menuë volaille ou gibier que ce puisse estre"; et "à ceux qui font profession d'entreprendre des festins de nôces, de fiançailles, ou des repas pour autres sujets, de prendre plus d'un écu par tête". Cependant, ajoute-t-il,

[c]ette Ordonnance est la derniere de nos Loix touchant les repas. L'usage depuis ce temps a changé. La France produit si abondamment toutes les choses necessaires à la vie, qu'il a esté jugé plus avantageux à ses Habitans, \& au bien de l'Estat de leur en laisser la libre disposition. C'est ce Commerce qui entretient dans les grandes Villes un nombre considerable d'Artisans, \& qui fait subsister la plus grande partie des Provinces 9 .

Les meilleurs esprits du premier $\mathrm{XVIII}^{\mathrm{e}}$ siècle se rendront à cet argument, qui est celui du mercantilisme libéral: Montesquieu, Voltaire refuseront l'équation du luxe et de la décadence; à rebours, la frugalité vieille-romaine prend sous leur plume le caractère de la régression. On se souvient de la Défense du mondain:

Sachez surtout que le luxe enrichit

Un grand État, s'il en perd un petit. [...]

J'entends d'ici des pédants à rabats,

Tristes censeurs des plaisirs qu'ils n'ont pas,

Qui, me citant Denys d'Halicarnasse,

Dion, Plutarque, et même un peu d'Horace,

Vont criaillant qu'un certain Curius,

9. Nicolas Delamare, Traité de la police, Paris, Jean \& Pierre Cot, 1705, t. I, p. 432. 
Cincinnatus et des consuls en us,

Bêchaient la terre au milieu des alarmes,

Qu'ils maniaient la charrue et les armes,

Et que les blés tenaient à grand honneur

D'être semés par la main d'un vainqueur. [...]

L'auguste Rome, avec tout son orgueil,

Rome, jadis, était ce qu'est Auteuil. [...]

Leur Jupiter, au temps du bon roi Tulle,

Était de bois; il fut d'or sous Luculle.

N'allez donc pas, avec simplicité,

Nommer vertu ce qui fut pauvreté ${ }^{10}$.

Le Mondain, lui, dans un décor peint par Poussin, boit du vin d'Aÿ et soupe à cinq services, après lesquels il hume, dans une tasse en porcelaine de Chine, les parfums d'un Mocha; l'on assiste ainsi, grâce au luxe, à une étrange interpénétration des deux mondes :

Le superflu, chose très nécessaire,

A réuni l'un et l'autre hémisphère.

Voyez-vous pas ces agiles vaisseaux

Qui du Texel, de Londres, de Bordeaux,

S'en vont chercher, par un heureux échange,

De nouveaux biens, nés aux sources du Gange,

Tandis qu'au loin, vainqueurs des musulmans,

Nos vins de France enivrent les sultans ${ }^{11}$ ?

Est-ce coïncidence si l'intérêt pour les peuples d'Orient connaît précisément à cette époque une si forte flambée? Les relations de Chardin (1686 et 1711) ou du chevalier d'Arvieux (1735); les Lettres édifiantes et curieuses sur la Chine écrites par les jésuites (1702-1776); la traduction des Mille et une nuits par Galland (1704-1717): tout cela a profondément changé la représentation que l'on s'était jusqu'alors fait de l'Oriental. Ce n'est plus un barbare. Bien qu'il vive sous un régime despotique et qu'il s'adonne un peu vivement aux plaisirs de la chair, ses mours sont naturellement bonnes. Le luxe et le raffinement dont il fait preuve, à table comme ailleurs, sont des marques de civilisation. Tandis que des ethnologues avant la lettre se penchent sur les usages épulaires exotiques - Antoine Galland, professeur d'arabe au Collège royal et fameux numismate, publie ainsi à Caen, en 1699, un

10. Voltaire, Défense du mondain ou L'apologie du luxe, dans Mélanges, éd. J. Van den Heuvel, Paris, Pléiade, 1961, p. 208-209.

11. Voltaire, ouvr. cité, p. 203. 
traité De l'origine et du progrez du café-12 — les auteurs d'ouvrages culinaires, dans leurs préfaces et leurs avertissements, font place à d'étonnants exposés "philosophiques" dus au concours de savants touche-à-tout: les jésuites Brumoy et Bougeant, Meusnier de Querlon, le polygraphe, de même que l'érudit Foncemagne rédigeront, sans les signer, les admirables morceaux d'entrée des Dons de Comus de François Marin (1739 et 1742) et de La science du maître d'bôtel cuisinier de Menon (1749), véritables pamphlets modernistes célébrant luxe, science et progrès.

La cuisine, comme tous les autres arts inventés pour le besoin ou pour le plaisir, s'est perfectionnée avec le génie des peuples, et elle est devenue plus délicate à mesure qu'ils se sont polis. Comme je n'entreprends pas d'en faire l'histoire, je ne m'arrêterai point à ses commencements. La vie des premiers hommes a dû ressembler à celle des peuples d'Amérique qui, bornés au simple nécessaire, ne pensaient point encore au superflu [...] Le luxe et la délicatesse de la table ont pris naissance dans l'Asie chez les Assyriens et les Perses, et la qualité du climat n'a pas peu contribué, sans doute, à rendre ces peuples si voluptueux ${ }^{13}$.

Il n'y a plus ici trace de censure: Brumoy et Bougeant rendent grâce aux Asiatiques de ce luxe qu'ils ont enseigné aux Grecs, qui de là s'est transmis aux Romains, puis aux Français par les Italiens - véritable translatio culinoe, indissociable du perfectionnement de l'homme, ce maître-mot de l'époque. En outre, assimilant la cuisine à la chimie, ils la faisaient bénéficier du prestige des sciences exactes et d'un matérialisme pour lequel esprit et corps sont homologues. De là à faire de l'art culinaire un facteur des Lumières, il n'y avait qu'un pas, franchi dix ans plus tard par Foncemagne:

La Cuisine subtilise les parties grossieres des alimens, dépouille les mixtes qu'elle employe, des sucs terrestres qu'ils contiennent: elle les perfectionne, les épure, \& les spiritualise en quelque sorte. Les mets qu'elle prépare, doivent donc porter dans le sang une plus grande abondance d'esprits plus purs \&

12. Antoine Galland, De l'origine et du progrez du café, sur un manuscrit arabe de la bibliothèque du Roy, Caen, Cavelier, Paris, Florentin et Delaulne, 1699.

13. Pierre Brumoy et Guillaume Hyacinthe Bougeant, "Avertissement ", dans François Marin, Dons de Comus [1739], éd. Béatrice Fink, Les liaisons savoureuses, Saint-Étienne, Publications de l'Université de Saint-Étienne, 1995, p. 26. 
plus déliés. De-là plus d'agilité \& de vigueur dans les corps, plus de vivacité \& de feu dans l'imagination, plus d'étendue \& de force dans le génie, plus de délicatesse \& de finesse dans nos goûts. Sera-ce donc trop s'avancer, que de placer les apprêts de la Cuisine moderne parmi les causes physiques qui du sein de la Barbarie, ont rappelé parmi nous le regne de la politesse, des talens de l'esprit, des Arts \& des Sciences ${ }^{14}$ ?

On constate avec amusement que cette "Dissertation préliminaire sur la Cusinine moderne" du très sérieux académicien paraît l'année même où Rousseau élabore son premier Discours, qui, reconnaissant l'union du luxe et du progrès, les honnissait tous deux. C'est que le vent tournait; la seconde moitié du siècle serait plus méfiante. Le chevalier de Jaucourt, dans l'article "Cuisine" de l'Encyclopédie, reprend, mais en mauvaise part, la translatio des deux jésuites, devenue contamination:

Les Asiatiques, plus voluptueux que les autres peuples, employerent les premiers, dans la préparation de leurs mets, toutes les productions de leurs climats; le commerce porta ces productions chez leurs voisins; l'homme courant après les richesses, n'en aima la joüissance que pour fournir à sa volupté, \& pour changer une simple \& bonne nourriture en d'autres plus abondantes, plus variées, plus sensuellement apprêtées, \& par conséquent plus nuisibles à la santé: c'est ainsi que la délicatesse des tables passa de l'Asie aux autres peuples de la terre. Les Perses communiquerent aux Grecs cette branche du luxe, à laquelle les sages législateurs de Lacédémone s'opposerent toûjours avec vigueur ${ }^{15}$.

Sans doute le médecin le dispute-t-il au philosophe chez Jaucourt; mais n'importe: le temps de l'optimisme anthropologique est passé. D'ailleurs, en phase avec un pythagorisme récemment mis à la mode, les Orientaux favoris de la fin du siècle seront les Indiens, sobres et végétariens, comme dans Les lettres d'Amabed (1769) de Voltaire.

Cependant revenons, pour conclure, à la rhétorique, cet autre membre de notre comparaison initiale. Tandis que la cuisine pre-

14. Étienne Lauréault de Foncemagne, "Dissertation préliminaire sur la Cuisine moderne", dans Menon, La science du mâ̂tre d'hôtel cuisinier, Paris, Paulusdu-Mesnil, 1749, p. xxii-xxiii.

15. Chevalier Louis de Jacucourt, article "Cuisine" de l'Encyclopédie, Paris, Briasson, David, Le Breton, Durand, 1754, t. IV, p. 537. 
nait son vol et gagnait en importance, l'antique ars dicendi a, au XVIII ${ }^{\mathrm{e}}$ siècle, son âge d'or derrière lui; quant à l'asianisme, il ne semble pas même qu'il ait survécu, du moins stricto sensu, à l'hégémonie du style naturel. À la fin du XviI ${ }^{\mathrm{e}}$ siècle, écrit Jean-Marie Valentin, la "connotation quasi universellement négative qui s'attache à l'enflure, au stylus inflatus [...], frappe tout ce qui se recommande encore de l'asianisme, de la Latinité d'argent, de l'engouement moderne enfin pour la recherche expressive qui étonne et surprend ${ }^{16}$ ". Et pourtant. Dans tout cet oriental fatras du siècle des Lumières, dans ces chinoiseries, dans ces turqueries qui évoquent parfois, à nos yeux fatigués, plus un boudoir de concierge qu'un sérail, une certaine forme d'asianisme ne s'estelle pas réfugiée? Loin des débats théoriques et des traités, chez cette fille de l'éloquence qui allait triompher sous le nom de littérature, on retrouve bien souvent les traits du style incriminé: abondance d'ornements, sensualité, variété imaginative; un luxe d'arabesques pas toujours innocent.

Ainsi, le destin parallèle que nous annoncions tout d'abord pour les arts du discours et ceux de la bonne chère semble se vérifier en ce début de $\mathrm{XVIII}^{\mathrm{e}}$ siècle et invite à penser simultanément, comme le voulaient Brumoy et Bougeant, une anthropologie morale et une esthétique. Au sein même des catégories du luxe et de la sobriété qui se maintiennent pourtant, on assiste à une inversion des valeurs traditionnelles: d'une part, le libéralisme économique et le sensationnisme font chorus pour chanter les plaisirs de la table; de l'autre, l'écriture libertine ou précieuse — chez Marivaux, chez Crébillon fils — s'accommode de l'efflorescence rococo des volutes et des courbes, et de la gaze qui voile les nudités trop franches ou les pensées trop hardies. D'ailleurs, l'ouverture militante des Philosophes au luxe et à l'Orient, aux voluptés de la grand'chère, à des morales neuves et fortes, tout cela impliquait un art de persuader sans doute moins déclaré que naguère, mais néanmoins efficace sous les oripeaux de la fiction. La rhétorique, au fond, n'est-elle pas chez eux la monnaie d'échange par laquelle on accède aux plaisirs interdits? Ils sont un peu comme ce porteur des Mille et une nuits qui, séduit par les

16. Jean-Marie Valentin, "De Leibniz à Vico. Contestation et restauration de la rhétorique (1690-1730)", dans Histoire de la rhétorique dans l'Europe moderne (1450-1950), sous la direction de M. Fumaroli, Paris, Presses universitaires de France, 1999, p. 830. 
32

apprêts d'un festin qu'allaient se donner trois beautés, entreprit de se faire inviter:

"Une compagnie de femmes sans hommes [dit-il] est pourtant une chose aussi triste qu'une compagnie d'hommes sans femmes." Il ajouta à ce discours plusieurs choses fort plaisantes pour prouver ce qu'il avançait. Il n'oublia pas de citer ce qu'on disait à Bagdad, qu'on n'est pas bien à table si l'on n'y est quatre; et enfin, il finit en concluant que puisqu'elles étaient trois, elles avaient besoin d'un quatrième. [...]. Zobéide connut que le porteur ne manquait pas d'esprit; mais jugeant qu'il avait envie d'être du régal qu'elles voulaient se donner, elle lui repartit en souriant: "Vous savez que nous nous préparons à nous régaler; mais vous savez en même temps que nous avons fait une dépense considérable, et il ne serait pas juste que, sans y contribuer, vous fussiez de la partie." [...]. Le porteur, malgré sa rhétorique, aurait peut-être été obligé de se retirer avec confusion, si Amine, prenant fortement son parti, n'eût dit à Zobéide et à Safie: "Mes chères sœurs, je vous conjure de permettre qu'il demeure avec nous: il n'est pas besoin de vous dire qu'il nous divertira; vous voyez bien qu'il en est capable " ${ }^{17}$.

Les gens de lettres impécunieux paient leur écot d'esprit, d'éloges et de contes. Leur goût du luxe aiguise leur ingéniosité; leur faim les rend "copieux". Un petit Oriental rusé se dissimule en chacun d'eux.

17. "Histoire des trois calenders fils de roi, et de cinq dames de Bagdad", dans Les mille et une nuits, trad. Antoine Galland, Paris, Garnier, 1988, vol. I, p. 79-80. 